Vol. 3, No. 1 | January - June 2020

\title{
Static Analysis of Six Bar Tensegrity Ball Structure Robot
}

\author{
Muhammad Basit Chandio ${ }^{1}$, Ani Luo ${ }^{1}$, Asif Raza ${ }^{1}$, Sanaullah Khushak ${ }^{1}$, \\ Muhammad Nazim Lakhan ${ }^{2}$, Altaf Hussain Shar ${ }^{2}$, Kishore Chand ${ }^{2}$, Fahad \\ Hussain $^{3}$, Zubair Ali Shah ${ }^{4}$
}

\begin{abstract}
:
All pre-stressed structures called as tensegrity structures have been introducing into robotics, modern architectural designs, and medical necessities called bio-tensegrity, space structure alternates, and many other emerging technologies because of its numerous useful properties. Six bar tensegrity ball robot structure has an essential importance in the field of robotics due to its deployable, movable, deformable, and easily controllable capabilities. This structure has 6 rigid bars (in compression) and 24 flexible strings (in tension) that are connected in such a 12 nodes arrangement that provides the highest spatial symmetrical shape to this structure among most of the tensegrity structure. In this paper, the internal forces of bars and strings on each node have been studied, and the force equation on every node has been developed. Furthermore, the balance of all bars and strings forces on the whole structure has been analytically verified to assure the structure remains statically pre-stressed under the zero net effect of all internal forces applied by bars and strings.
\end{abstract}

Keywords: static analysis; force equations; equilibrium

\section{Introduction}

Tensegrity structures are formed by a combination of rigid elements (the bars) and elastic elements (the strings) [1-4]. The bars are always in compression and the strings in tension. The entire structure stands by itself and maintains its form solely because of the internal arrangement of the strings and the bars [5-8]. Tensegrity structures were used only in arts and architecture in the early1960s when fuller coined this tensegrity idea [9-12]. Orthopedic surgeon Stephen Levin found a tensegrity structure could be a new biomechanical approach and coined a biotensegrity term in the medical field in the 1970s[13], later these structures have been remaining a vital part in medical researches[14-17]. Tensegrity structures are also used in robotics because of implement flexibility, dynamism, and light-weighting

${ }^{1}$ College of Mechanical \& Electrical Engineering, Harbin Engineering University, Harbin, China

${ }^{2}$ College of Material Science and Chemical Engineering, Harbin Engineering University, Harbin, China

${ }^{3}$ Department of Mechanical Engineering MUET SZAB Campus Khairpur Mir's, Pakistan

${ }^{4}$ College of Power \& Energy Engineering, Harbin Engineering University, Harbin, China

Corresponding Author: basitchandio47@ gmail.com

$$
\text { SJET | ISSN: 2616-7069 | Vol. } 3 \text { | No. } 1 \text { | @ } 2020 \text { Sukkur IBA University }
$$


[18-21]. These structures are also used in space applications due to super deplorability $[22,23]$.

Louani modeled the mathematics of the six-bar tensegrity ball structure robot[24] and analyzed the possible and feasible ways to drive this structure [25-27]. NASA proposed a six-bar tensegrity ball robot structure as workable in landing on different surfaces for planetary exploration missions and easy to move and control the structure [28, 29].

It is vital to recognize the all internal forces exerted by all bars and strings on each node of six-bar tensegrity ball structure robot in a static position in order to move it by servomotors or to land it for planetary exploring mission to protect the payload. Skelton in his book and Julio in his paper have mentioned basic general methods to analyze the static tensegrity structures $[30,31]$. But, there is a gap of static analysis of the six-bar tensegrity ball structure robot in research. Here, a static study of the six-bar tensegrity ball structure has been done to analyze the position and forces exerted by all bars and strings on each node and the overall net effect of all internal forces of the structure has been observed to verify the static mode of structure.

In this paper, a six-bar tensegrity ball structure robot has been defined and a node matrix has been developed. Later, Individual force equations for all nodes have been established. In the last, all individual force equations on each node have been examined to validate the static position of the structure by balancing the bars forces and strings forces.

\section{Introduction of the Six Bar Tensegrity Ball Robot Structure}

Six bar tensegrity ball robot structure has 6 rigid bars in compression and 24 flexible strings in tension. All bars are shown by blue color and strings are shown by red color and are connected by 12 nodes. Each node is connected with one bar and four strings. In this structure, all six bars are lying along three different planes. Each plane has two parallel bars. Bars $\boldsymbol{b}_{\boldsymbol{1}} \& \boldsymbol{b}_{\boldsymbol{2}}$ lay along $X Y$ plane, bars $\boldsymbol{b}_{\mathbf{3}} \& \boldsymbol{b}_{\mathbf{4}}$ lay along $Y Z$ plane and bars $\boldsymbol{b}_{5} \& \boldsymbol{b}_{\boldsymbol{6}}$ lay along $X Z$ plane. Similarly nodes 1,2,3 \& 4 are in $X Y$ plane, nodes 5,6,7 $\& 8$ are in $Y Z$ plane and nodes $9,10,11 \& 12$ are in $X Z$ plane as illustrated in Fig.1.
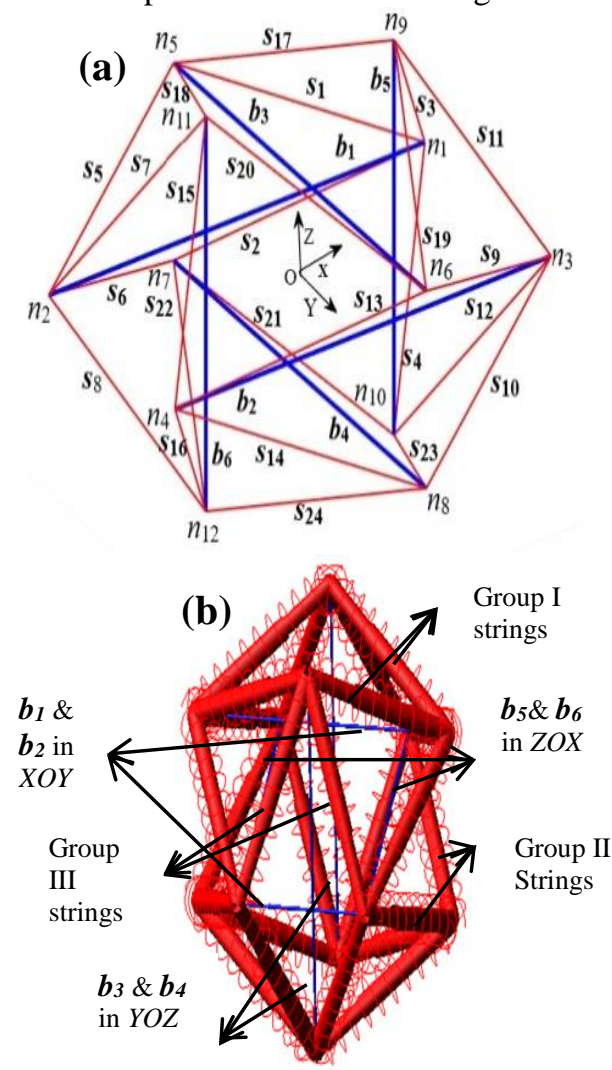

Fig. 1. Six bar tensegrity ball structure robot illustrated (a) in MATLAB \& (b) in ADAMS

All members of six-bar tensegrity structure are connected by 12 nodes and a node $n_{i}$ can be represented in Cartesian coordinates as

$$
n_{i}=\left[\begin{array}{lll}
n_{i x} & n_{i y} & n_{i z}
\end{array}\right], i \in[1,12]
$$


Node matrix $(\mathrm{N})$ can be obtained by putting all nodes in a column of the matrix.

$$
\mathbf{N}=\left[n_{1}, n_{2}, n_{3} \ldots n_{12}\right]_{3 \times 12}
$$

Coordinates of all 12 nodes along three axes can be rearranged as displayed in Eq. (1) [24].

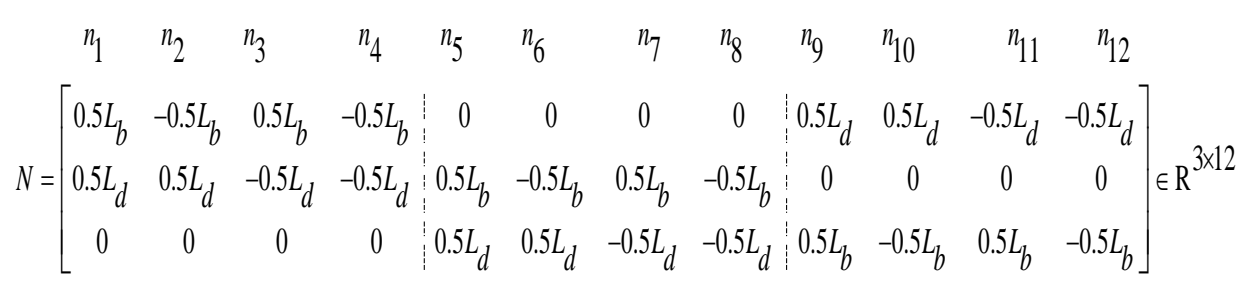

\section{Internal Forces on Structure}

Every node of the six-bar tensegrity ball structure is acted by four strings forces and one bar force. We have modeled separate equations of forces for each node in all three axes. Force density in strings is the tension applied string per unit its length (i-e: $\boldsymbol{\gamma}=\boldsymbol{T}_{s} / \boldsymbol{L}_{\boldsymbol{s}}$ ) and force density by the bar is the force applied by bar per unit its length (i-e: $\left.\boldsymbol{\lambda}=\boldsymbol{F}_{\boldsymbol{b}} / \boldsymbol{L}_{\boldsymbol{b}}\right)[24,30]$. Six bar tensegrity structure is spatial structure and lies along three space axes. Each plane contains four nodes.

\subsection{Internal Forces on Nodes laying along $X Y$ Plane}

Plane $X Y$ contains four nodes $1,2,3 \& 4$. We find the force equations of each node. Here, node 1 is connected with strings $\boldsymbol{s}_{1}, \boldsymbol{s}_{2}$, $\boldsymbol{s}_{3}, \boldsymbol{s}_{4} \&$ bar $\boldsymbol{b}_{1}$ in such an arrangement that strings pull the node inwards and bar pushes it back by the forces $\boldsymbol{T}_{s 1}, \boldsymbol{T}_{\boldsymbol{s} 2}, \boldsymbol{T}_{\mathbf{s} 3}, \boldsymbol{T}_{\boldsymbol{s} 4} \& \boldsymbol{F}_{\boldsymbol{b} 1}$ respectively, but the node does not move in any direction as shown in Fig. 2 .

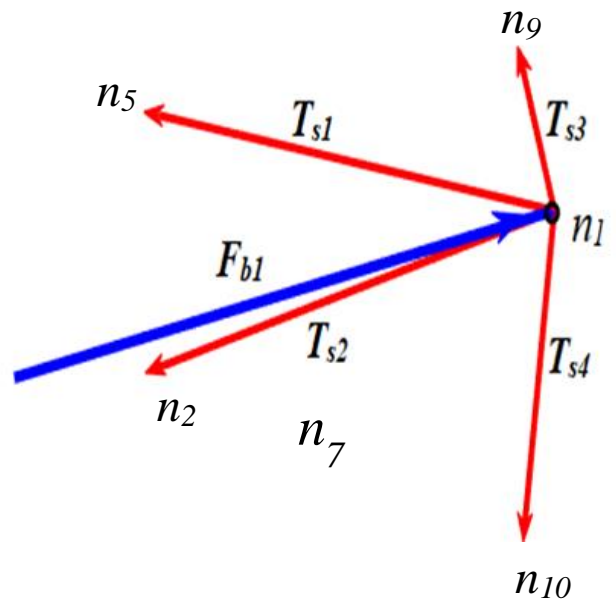

Fig. 2. Forces on node 1

$$
F_{b_{1}}+T_{s_{1}}+T_{s_{2}}+T_{s_{3}}+T_{s_{4}}=0
$$

As we already mentioned $\boldsymbol{\lambda}_{\boldsymbol{1}} \boldsymbol{F}_{\boldsymbol{b}} / \boldsymbol{L}_{\boldsymbol{b}} \quad \&$ $\gamma=\boldsymbol{T}_{s} / \boldsymbol{L}_{\boldsymbol{s}}$ in section 3 , therefore, by putting substitutes of bar force and all string forces in terms of force densities in eq. (2), we achieve 


$$
\lambda_{1}\left(\mathrm{n}_{1}-n_{2}\right)+\gamma_{1}\left(\mathrm{n}_{5}-n_{1}\right)+\gamma_{2}\left(\mathrm{n}_{7}-n_{1}\right)+\gamma_{3}\left(\mathrm{n}_{9}-n_{1}\right)+\gamma_{4}\left(\mathrm{n}_{10}-n_{1}\right)=0
$$

All bars have the same force density as $\lambda$, and all strings have the same force density as $\gamma$ when no external force acts on the structure.

$$
\begin{gathered}
\lambda_{1}\left(\mathrm{n}_{2}-n_{1}\right)+\gamma_{5}\left(\mathrm{n}_{5}-n_{2}\right)+\gamma_{6}\left(\mathrm{n}_{7}-n_{2}\right)+\gamma_{7}\left(\mathrm{n}_{11}-n_{2}\right)+\gamma_{8}\left(\mathrm{n}_{12}-n_{2}\right)=0 \\
\lambda\left[\begin{array}{c}
n_{1 x}-n_{2 x} \\
n_{1 y}-n_{2 y} \\
n_{1 z}-n_{2 z}
\end{array}\right]+\gamma\left[\begin{array}{c}
n_{5 x}+n_{7 x}+n_{9 x}+n_{10 x}-4 n_{1 x} \\
n_{5 y}+n_{7 y}+n_{9 y}+n_{10 y}-4 n_{1 y} \\
n_{5 z}+n_{7 z}+n_{9 z}+n_{10 z}-4 n_{1 z}
\end{array}\right]=0
\end{gathered}
$$

We get Eq. (4) by putting coordinates of nodes in Eq. (3)

$$
\lambda\left[\begin{array}{c}
0.5 L_{b}-\left(-0.5 L_{b}\right) \\
0.5 L_{d}-0.5 L_{d} \\
0-0
\end{array}\right]+\gamma\left[\begin{array}{c}
0+0+0.5 L_{d}+0.5 L_{d}-4 \times 0.5 L_{b} \\
0.5 L_{b}+0.5 L_{b}+0+0-4 \times 0.5 L_{d} \\
0.5 L_{d}+\left(-0.5 L_{d}\right)+0.5 L_{b}+\left(-0.5 L_{b}\right)-4 \times 0
\end{array}\right]=0
$$

Force equations along the $\mathrm{x}$-axis and $\mathrm{y}$-axis on node 1 are Eq. (5) \& Eq. (6) respectively and force equation along the $\mathrm{z}$-axis is zero.

$$
\begin{aligned}
& \lambda\left(\mathrm{L}_{b}\right)+\gamma\left(L_{d}-2 L_{b}\right)=0 \\
& \gamma\left(L_{b}-2 L_{d}\right)=0
\end{aligned}
$$

Eq. (5) \& Eq. (6) are force equations in terms of force densities, bars length, and the perpendicular distance between two parallel bars.

Similarly, we find force equations on node 2 . this node is connected with strings $\boldsymbol{s}_{\boldsymbol{5}}$, $\boldsymbol{S}_{6}, \boldsymbol{S}_{7}, \boldsymbol{s}_{8} \&$ bar $\boldsymbol{b}_{1}$ in such an arrangement that strings pull the node inwards and bar pushes it back by forces $\boldsymbol{T}_{s 5}, \boldsymbol{T}_{s 6}, \boldsymbol{T}_{s}, \boldsymbol{T}_{s 8} \& \boldsymbol{F}_{b 1}$ respectively, but this node does not move in any direction also as illustrated in Fig. 3.

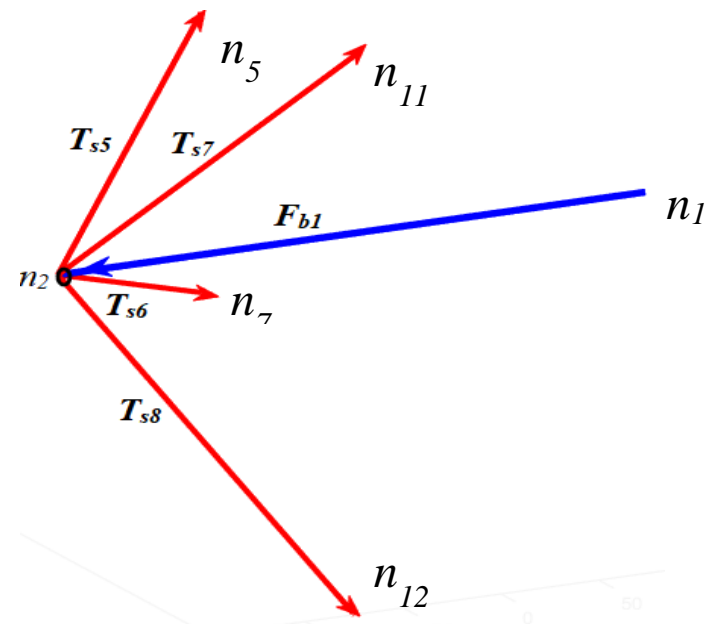

Fig 3. Forces on node 2 


$$
F_{b_{1}}+T_{s_{5}}+T_{s_{6}}+T_{S_{7}}+T_{S_{8}}=0
$$

Similarly, by putting substitutes of bar force and all string forces in terms of force densities eq. (7), we achieve

$$
\lambda_{1}\left(\mathrm{n}_{2}-n_{1}\right)+\gamma_{5}\left(\mathrm{n}_{5}-n_{2}\right)+\gamma_{6}\left(\mathrm{n}_{7}-n_{2}\right)+\gamma_{7}\left(\mathrm{n}_{11}-n_{2}\right)+\gamma_{8}\left(\mathrm{n}_{12}-n_{2}\right)=0
$$

All bars have the same force density as $\lambda$, and all strings have the same force density as $\gamma$ when no external force acts on structure, therefore

$$
\begin{gathered}
\lambda\left(n_{2}-n_{1}\right)+\gamma\left(n_{5}+n_{7}+n_{11}+n_{12}-4 n_{2}\right)=0 \\
\lambda\left[\begin{array}{l}
n_{2 x}-n_{1 x} \\
n_{2 y}-n_{1 y} \\
n_{2 z}-n_{1 z}
\end{array}\right]+\gamma\left[\begin{array}{c}
n_{5 x}+n_{7 x}+n_{11 x}+n_{12 x}-4 n_{2 x} \\
n_{5 y}+n_{7 y}+n_{11 y}+n_{12 y}-4 n_{2 y} \\
n_{5 z}+n_{7 z}+n_{11 z}+n_{12 z}-4 n_{2 z}
\end{array}\right]=0
\end{gathered}
$$

We get Eq. (9) by putting coordinates of nodes in Eq. (8)

$$
\lambda\left[\begin{array}{c}
\left(-0.5 L_{b}\right)-0.5 L_{b} \\
0.5 L_{d}-0.5 L_{d} \\
0-0
\end{array}\right]+\gamma\left[\begin{array}{c}
0+0+\left(-0.5 L_{d}\right)+\left(-0.5 L_{d}\right)-4 \times\left(-0.5 L_{b}\right) \\
0.5 L_{b}+0.5 L_{b}+0+0-4 \times 0.5 L_{d} \\
0.5 L_{d}+\left(-0.5 L_{d}\right)+0.5 L_{b}+\left(-0.5 L_{b}\right)-4 \times 0
\end{array}\right]=0
$$

Force equations along the $\mathrm{x}$-axis and $\mathrm{y}$ axis on node 2 are in Eq. (10) \& Eq. (11) respectively and force equation along the $\mathrm{z}$ axis is zero

$$
\begin{gathered}
\lambda\left(-L_{b}\right)+\gamma\left(-L_{d}+2 L_{b}\right)=0 \\
\gamma\left(L_{b}-2 L_{d}\right)=0
\end{gathered}
$$

Eq. (10) \& Eq. (11) are force equations in terms of force densities, bars length, and the perpendicular distance between two parallel bars. Again, node 3 is connected with strings $\boldsymbol{s}_{\boldsymbol{1}}, \boldsymbol{s}_{10}, \boldsymbol{s}_{11}, \boldsymbol{s}_{12} \&$ bar $\boldsymbol{b}_{2}$ in such an arrangement that strings pull the node inwards and bar pushes it back by forces $\boldsymbol{T}_{s} 9, \boldsymbol{T}_{s 10}, \boldsymbol{T}_{s 11}, \boldsymbol{T}_{s 12} \&$
$\boldsymbol{F}_{\boldsymbol{b} 2}$ respectively, but this node also does not move in any direction as shown in Fig. 4.

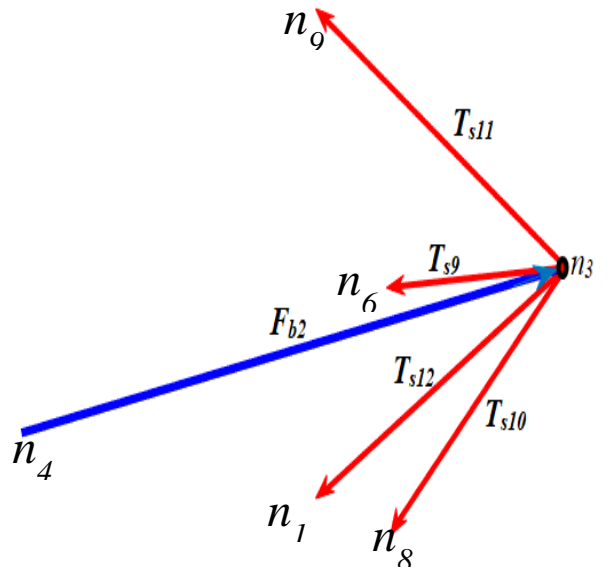

Fig. 4. Forces on node 3 


$$
F_{b_{2}}+T_{S_{9}}+T_{s_{10}}+T_{S_{11}}+T_{s_{12}}=0
$$

Similarly, by putting substitutes of bar force and all string forces in terms of force densities eq. (12), we achieve

$$
\lambda_{2}\left(n_{3}-n_{4}\right)+\gamma_{9}\left(n_{6}-n_{3}\right)+\gamma_{10}\left(n_{8}-n_{3}\right)+\gamma_{11}\left(n_{9}-n_{3}\right)+\gamma_{12}\left(n_{10}-n_{3}\right)=0
$$

All bars have the same force density as $\lambda$ and all strings have the same force density as $\gamma$ when no external force acts on structure, therefore

$$
\begin{gathered}
\lambda\left(n_{3}-n_{4}\right)+\gamma\left(n_{6}+n_{8}+n_{9}+n_{10}-4 n_{3}\right)=0 \\
\lambda\left[\begin{array}{c}
n_{3 x}-n_{4 x} \\
n_{3 y}-n_{4 y} \\
n_{3 z}-n_{4 z}
\end{array}\right]+\gamma\left[\begin{array}{c}
n_{6 x}+n_{8 x}+n_{9 x}+n_{10 x}-4 n_{3 x} \\
n_{6 y}+n_{8 y}+n_{9 y}+n_{10 y}-4 n_{3 y} \\
n_{6 z}+n_{8 z}+n_{9 z}+n_{10 z}-4 n_{3 z}
\end{array}\right]=0
\end{gathered}
$$

We get Eq. (14) by putting coordinates of nodes in Eq. (13)

$$
\lambda\left[\begin{array}{c}
0.5 L_{b}-\left(-0.5 L_{b}\right) \\
-0.5 L_{d}-\left(-0.5 L_{d}\right) \\
0-0
\end{array}\right]+\gamma\left[\begin{array}{c}
0+0+0.5 L_{d}+0.5 L_{d}-4 \times\left(0.5 L_{b}\right) \\
-0.5 L_{b}+\left(-0.5 L_{b}\right)+0+0-4 \times\left(-0.5 L_{d}\right) \\
0.5 L_{d}+\left(-0.5 L_{d}\right)+0.5 L_{b}+\left(-0.5 L_{b}\right)-4 \times 0
\end{array}\right]=0
$$

Force equations along the $\mathrm{x}$-axis and $\mathrm{y}$ axis on node 3 are in Eq. (15) \& Eq. (16) respectively and force equation along the $\mathrm{z}$ axis is zero

$$
\begin{gathered}
\lambda\left(L_{b}\right)+\gamma\left(L_{d}-2 L_{b}\right)=0 \\
\gamma\left(-L_{b}+2 L_{d}\right)=0
\end{gathered}
$$

Eq. (15) \& Eq. (16) are force equations in terms of force densities, bars length, and the perpendicular distance between two parallel bars.

Likewise, node 4 is connected with strings $s_{13}, s_{14}, s_{15}, s_{16} \&$ bar $b_{2}$ in such an arrangement that strings pull the node inwards and bar pushes it back by forces $\boldsymbol{T}_{\boldsymbol{s} 13}$, $\boldsymbol{T}_{s 14}, \boldsymbol{T}_{\boldsymbol{s} 15}, \boldsymbol{T}_{s 16} \& \boldsymbol{F}_{\boldsymbol{b} 2}$ respectively, but the node does not move in any direction as illustrated in Fig. 5.

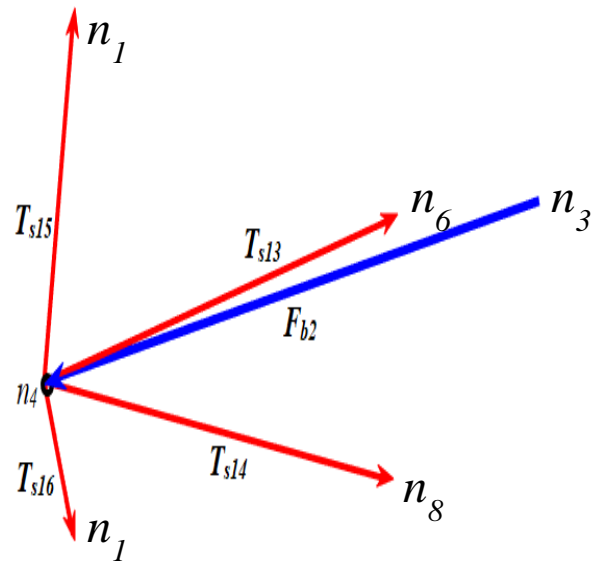

Fig 5. Forces on node 4 


$$
\begin{gathered}
F_{b_{2}}+T_{s_{13}}+T_{s_{14}}+T_{s_{15}}+T_{s_{16}}=0 \quad \text { (17) } \begin{array}{r}
\text { Similarly, by putting substitutes of bar } \\
\text { force and all string forces in terms of force } \\
\text { densities eq. }(17), \text { we achieve }
\end{array} \\
\lambda_{2}\left(n_{4}-n_{3}\right)+\gamma_{13}\left(n_{6}-n_{4}\right)+\gamma_{14}\left(n_{8}-n_{4}\right)+\gamma_{14}\left(n_{11}-n_{4}\right)+\gamma_{15}\left(n_{12}-n_{4}\right)=0
\end{gathered}
$$

All bars have the same force density as $\lambda$, and all strings have the same force density as $\gamma$ when no external force acts on structure, therefore

$$
\begin{gathered}
\lambda\left(n_{4}-n_{3}\right)+\gamma\left(n_{6}+n_{8}+n_{11}+n_{12}-4 n_{4}\right)=O \\
\lambda\left[\begin{array}{c}
n_{4 x}-n_{3 x} \\
n_{4 y}-n_{3 y} \\
n_{4 z}-n_{3 z}
\end{array}\right]+\gamma\left[\begin{array}{c}
n_{6 x}+n_{8 x}+n_{11 x}+n_{12 x}-4 n_{4 x} \\
n_{6 y}+n_{8 y}+n_{11 y}+n_{12 y}-4 n_{4 y} \\
n_{6 z}+n_{8 z}+n_{11 z}+n_{12 z}-4 n_{4 z}
\end{array}\right]=0
\end{gathered}
$$

We get Eq. (19) by putting coordinates of nodes in Eq. (18)

$$
\lambda\left[\begin{array}{c}
-0.5 L_{b}-0.5 L_{b} \\
-0.5 L_{d}-\left(-0.5 L_{d}\right) \\
0-0
\end{array}\right]+\gamma\left[\begin{array}{c}
0+0-0.5 L_{d}-0.5 L_{d}-4 \times\left(-0.5 L_{b}\right) \\
-0.5 L_{b}+\left(-0.5 L_{b}\right)+0+0-4 \times\left(-0.5 L_{d}\right) \\
0.5 L_{d}+\left(-0.5 L_{d}\right)+0.5 L_{b}+\left(-0.5 L_{b}\right)-4 \times 0
\end{array}\right]=0
$$

Force equations along the $\mathrm{x}$-axis and $\mathrm{y}$ axis on node 4 are in Eq. (20) \& Eq. (21) respectively and force equation along the $\mathrm{z}$ axis is zero

$$
\begin{aligned}
& \lambda\left(-L_{b}\right)+\gamma\left(-L_{d}+2 L_{b}\right)=0 \\
& \gamma\left(-L_{b}+2 L_{d}\right)=0
\end{aligned}
$$

Eq. (20) \& Eq. (21) are force equations in terms of force densities, bars length, and the perpendicular distance between two parallel bars.

\subsection{Internal Forces on Nodes laying along YZ Plane}

Plane $Y Z$ also contains four nodes 5, 6, 7 $\& 8$. Here in this section, we just put the force equations on each node lying in the $Y Z$ plane, and these equations can be found with the same method used in section 3.1.

Here, node 5 is connected with four strings $\boldsymbol{s}_{1}, \boldsymbol{s}_{5}, \boldsymbol{s}_{17}, \boldsymbol{s}_{18} \&$ one bar $\boldsymbol{b}_{3}$ in such an arrangement ${ }^{20)}$ that strings pull the node inwards and par pushes it back by forces $\boldsymbol{T}_{\boldsymbol{s}}$, $\boldsymbol{T}_{\boldsymbol{s} 5}, \boldsymbol{T} \boldsymbol{s} 17, \boldsymbol{T}_{\boldsymbol{s} 18} \& \boldsymbol{F}_{\boldsymbol{b} 3}$ respectively, but the node does not move in any direction as shown in Fig. 6

$$
F_{b_{3}}+T_{s_{1}}+T_{s_{5}}+T_{s_{17}}+T_{s_{18}}=0
$$

We found force equations in terms of force densities, bars length, and the perpendicular distance between two parallel bars along the $y$-axis and $\mathrm{z}$-axis on node 5 in 
Eq. (23) \& Eq. (24) respectively and force equation along the $\mathrm{x}$-axis as zero.

$$
\begin{gathered}
\lambda\left(L_{b}\right)+\gamma\left(L_{d}-2 L_{b}\right)=0 \\
\gamma\left(L_{b}-2 L_{d}\right)=0
\end{gathered}
$$

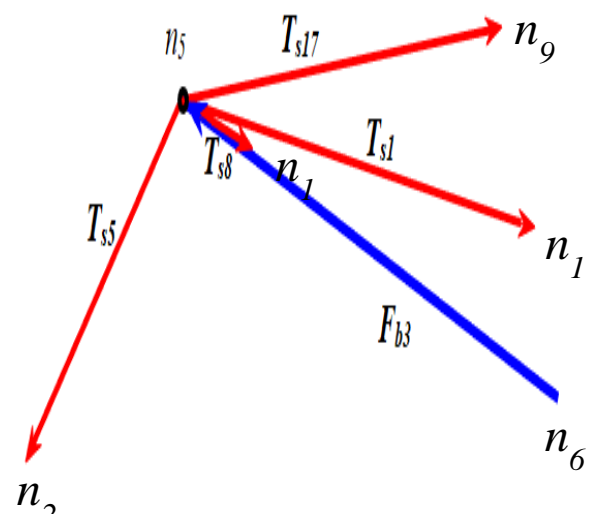

Fig. 6. Forces on node 5

Similarly, $\boldsymbol{T}_{s}$, $, \boldsymbol{T}_{\boldsymbol{s 1 3}}, \boldsymbol{T}_{\boldsymbol{s} 19}, \boldsymbol{T}_{\boldsymbol{s} 20} \& \boldsymbol{F}_{\boldsymbol{b 3}}$ are forces exerted by four strings $s_{9}, s_{13}, s_{19}, s_{20}$ $\&$ one bar $\boldsymbol{b}_{3}$ on node 6 in such manner that strings pull the node inwards and bar pushes it back and the node does not move in any direction as shown in Fig. 7.

$F_{b_{3}}+T_{s_{9}}+T_{s_{13}}+T_{s_{19}}+T_{s_{20}}=0$

We found force equations in terms of force densities, bars length, and the perpendicular distance between two parallel bars along the $y$-axis and z-axis on node 6 as in Eq. (26) \& Eq. (27) respectively and force equation along the $\mathrm{x}$-axis as zero.

$$
\begin{gathered}
\lambda\left(-L_{b}\right)+\gamma\left(-L_{d}+2 L_{b}\right)=0 \\
\gamma\left(L_{b}-2 L_{d}\right)=0
\end{gathered}
$$

Four strings $s_{2}, s_{6}, s_{21}, s_{22}, \&$ one bar $b_{4}$ exert forces $\boldsymbol{T}_{\boldsymbol{s} 2}, \boldsymbol{T}_{\boldsymbol{s} \boldsymbol{6}}, \boldsymbol{T}_{\boldsymbol{s} 21}, \boldsymbol{T}_{\boldsymbol{s} 22} \& \boldsymbol{F}_{\boldsymbol{b} 4}$ respectively one node 7 in such manner that this node has no movement in any direction as shown in Fig. 8.

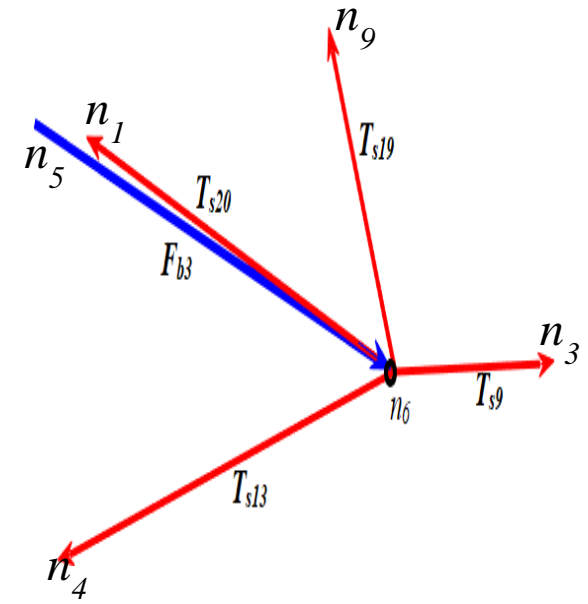

Fig. 7. Forces on node 6

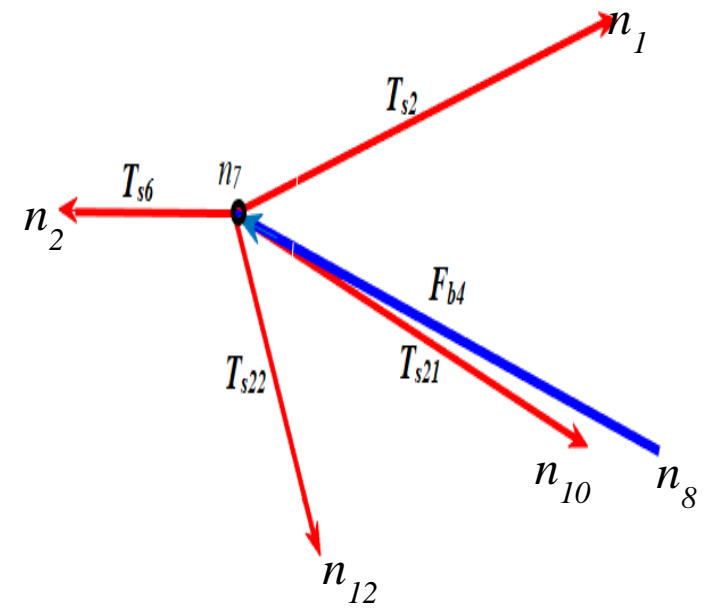

Fig. 8. Forces on node 7

$$
F_{b_{4}}+T_{s_{2}}+T_{s_{6}}+T_{s_{21}}+T_{s_{22}}=0
$$


We found force equations in terms of force densities, bars length, and the perpendicular distance between two parallel bars along the $y$-axis and $z$-axis on node 7 as in Eq. (29) \& Eq. (30). Moreover, the force equation along the $\mathrm{x}$-axis is zero.

$$
\begin{gathered}
\lambda\left(L_{b}\right)+\gamma\left(L_{d}-2 L_{b}\right)=0 \\
\gamma\left(-L_{b}+2 L_{d}\right)=0
\end{gathered}
$$

Similarly, node 8 is balanced by forces $\boldsymbol{T}_{s 10}, \boldsymbol{T}_{s 14}, \boldsymbol{T}_{s 23}, \boldsymbol{T}_{s 24} \& \boldsymbol{F}_{b 4}$ applied by four strings $s_{10}, s_{14}, s_{23}, s_{24} \&$ one bar $b_{4}$ as shown in Fig.9.

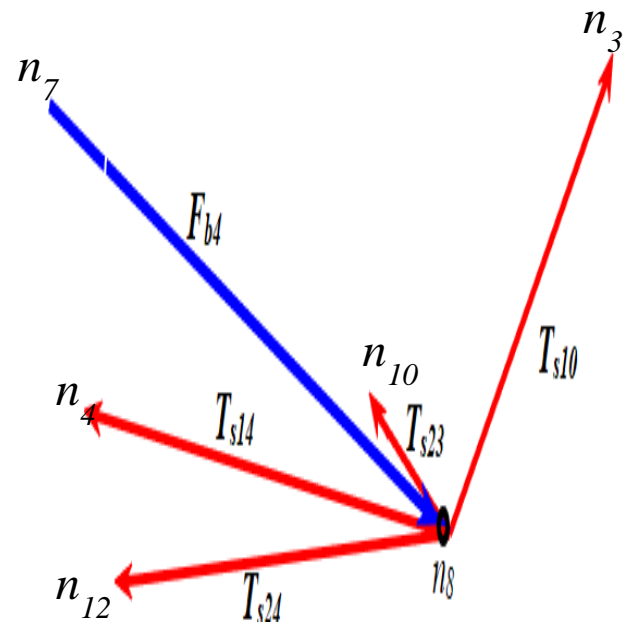

Fig. 9. Forces on node 8

$$
F_{b_{4}}+T_{S_{10}}+T_{S_{14}}+T_{S_{23}}+T_{S_{24}}=0
$$

We found force equations in terms of force densities, bars length, and the perpendicular distance between two parallel bars along the y-axis and z-axis on node 8 as in Eq. (32) \& Eq. (33) respectively and force equation along the $\mathrm{x}$-axis is zero.

$$
\lambda\left(-L_{b}\right)+\gamma\left(-L_{d}+2 L_{b}\right)=0
$$

$$
\gamma\left(-L_{b}+2 L_{d}\right)=0
$$

\subsection{Internal Forces on Nodes laying along $X Z$ Plane}

Plane $X Z$ also contains four nodes 9, 10, $11 \& 12$. Here in this section, we just put the force equations on each node lying in the $X Z$ plane, and these equations can be found with the same method used in section 3.1.

So, node 9 is connected with four strings $s_{10}, s_{14}, s_{23}, s_{24} \&$ one bar $b_{5}$ in such an arrangement that strings pull the node inwards and bar pushes it back by the forces $\boldsymbol{T}_{s 10}, \boldsymbol{T}_{s 14}, \boldsymbol{T}_{s 23}, \boldsymbol{T}_{s 24} \& \boldsymbol{F}_{b 5}$ respectively but node remains in the same position as shown in Fig.10.

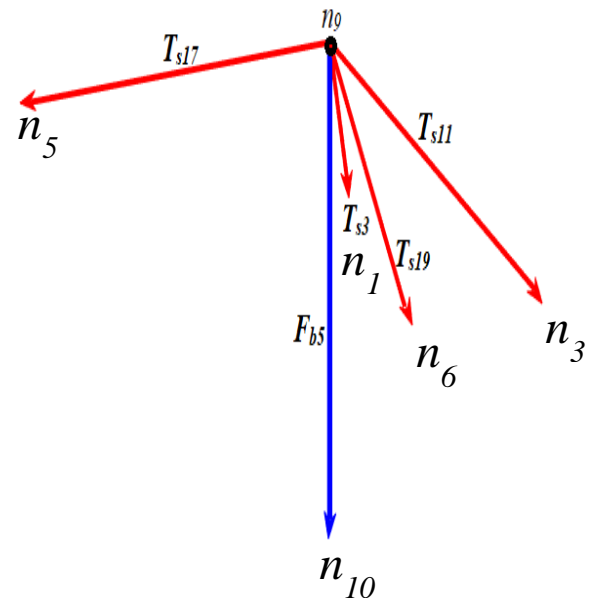

Fig 10. Forces on node 9

$F_{b_{5}}+T_{s_{3}}+T_{s_{11}}+T_{s_{17}}+T_{s_{19}}=0$

We found force equations in terms of force densities, bars length, and the perpendicular distance between two parallel bars along the $\mathrm{x}$-axis and $\mathrm{z}$-axis on node 9 as 
in Eq. (35) \& Eq. (36) respectively and force equation along the $\mathrm{y}$-axis as zero.

$$
\begin{gathered}
\gamma\left(L_{b}-2 L_{d}\right)=0 \\
\lambda\left(L_{b}\right)+\gamma\left(L_{d}-2 L_{b}\right)=0
\end{gathered}
$$

Similarly, four strings $s_{4}, s_{12}, s_{21}, s_{23}, \&$ one bar $\boldsymbol{b}_{5}$ apply forces $\boldsymbol{T}_{s 4}, \boldsymbol{T}_{\boldsymbol{s} 12}, \boldsymbol{T}_{\boldsymbol{s} 21}, \boldsymbol{T}_{\boldsymbol{s} 23} \&$ $\boldsymbol{F}_{\boldsymbol{b} 5}$ respectively on node 10 in such manner that node does not move in any direction as shown in Fig. 11.

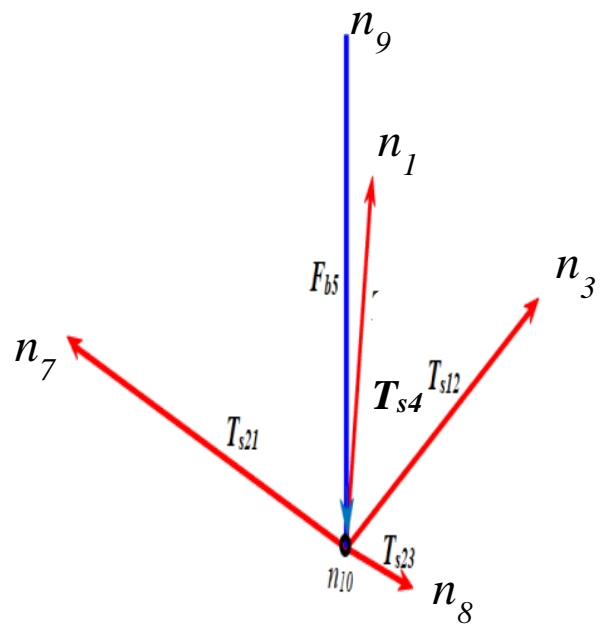

Fig. 11. Forces on node 10

$F_{b_{5}}+T_{s_{4}}+T_{s_{12}}+T_{s_{21}}+T_{s_{23}}=0$

We found force equations in terms of force densities, bars length, and the perpendicular distance between two parallel bars along the $\mathrm{x}$-axis and $\mathrm{z}$-axis on node 10 as in Eq. (38) \& Eq. (39) respectively and force equation along the $y$-axis as zero.

$$
\gamma\left(L_{b}-2 L_{d}\right)=0
$$

$$
\lambda\left(-L_{b}\right)+\gamma\left(-L_{d}+2 L_{b}\right)=0
$$

Node 11 is connected with strings $s_{7}, s_{15}$, $\boldsymbol{s}_{18}, \boldsymbol{s}_{20}, \& \boldsymbol{b}_{6}$ in such an arrangement that strings pull the node inwards and bar pushes it back $\boldsymbol{T}_{s 7}, \boldsymbol{T}_{\boldsymbol{s} 15}, \boldsymbol{T}_{\boldsymbol{s} 18}, \boldsymbol{T}_{\boldsymbol{s} 20} \& \boldsymbol{F}_{\boldsymbol{b} 6}$ respectively, but the node does not move in any direction as illustrated in Fig. 12.

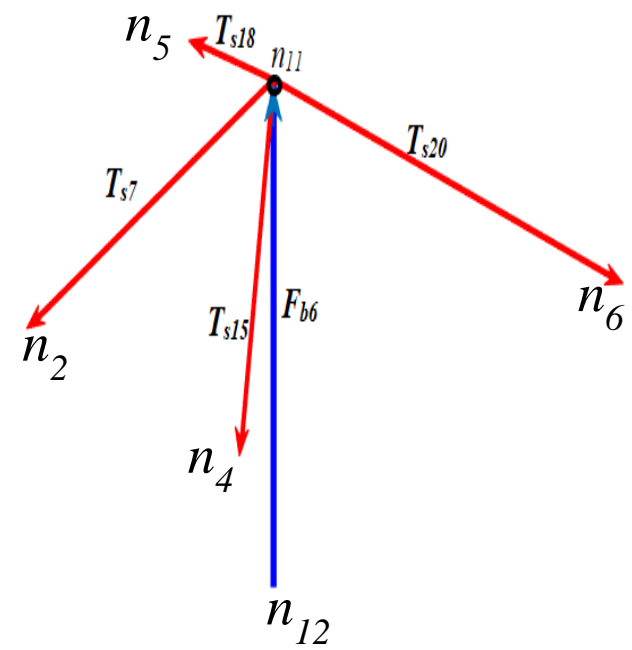

Fig. 12. Forces on node 11

$$
F_{b_{6}}+T_{s_{7}}+T_{s_{15}}+T_{s_{18}}+T_{s_{20}}=0
$$

We found force equations in terms of force densities, bars length, and the perpendicular distance between two parallel bars along the $\mathrm{x}$-axis and $\mathrm{z}$-axis on node 11 as in Eq. (41) \& Eq. (42) respectively and force equation along the $\mathrm{y}$-axis as zero.

$$
\begin{gathered}
\gamma\left(-L_{b}+2 L_{d}\right)=0 \\
\lambda\left(L_{b}\right)+\gamma\left(L_{d}-2 L_{b}\right)=0
\end{gathered}
$$

Finally, node 12 is pulled by forces $\boldsymbol{T}_{\boldsymbol{s}}$, $T_{s 16}, T_{s 22}, T_{s 24}$ applied by four strings $S_{8}, \boldsymbol{S}_{16}$, $\boldsymbol{S}_{22}, \boldsymbol{S}_{24}$, and pushed back by force $\boldsymbol{F}_{\boldsymbol{b} \boldsymbol{6}}$ applied 
by bar $\boldsymbol{b}_{\boldsymbol{6}}$, but the node does not experience any movement in any direction as shown in Fig.13.

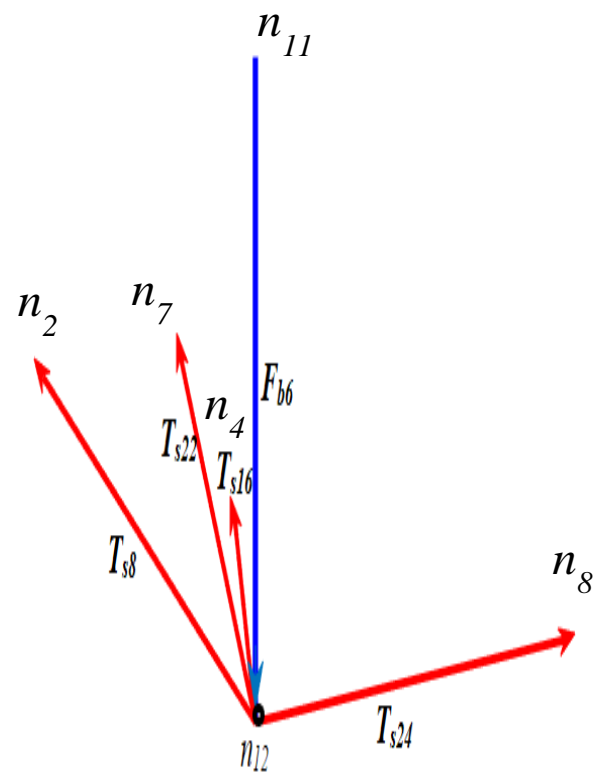

Fig. 13. Forces on node 12
$F_{b_{6}}+T_{s_{8}}+T_{s_{16}}+T_{s_{22}}+T_{s_{24}}=0$

We found force equations in terms of force densities, bars length, and the perpendicular distance between two parallel bars along the $\mathrm{x}$-axis and $\mathrm{z}$-axis on node 12 , as in Eq. (44) \& Eq. (45) respectively and force equation along the $y$-axis as zero.

$$
\begin{gathered}
\gamma\left(-L_{b}+2 L_{d}\right)=0 \\
\lambda\left(-L_{b}\right)+\gamma\left(-L_{d}+2 L_{b}\right)=0
\end{gathered}
$$

\subsection{Internal force on the whole structure}

We have found force equations of all 12 nodes along three axes individually. Here, we add all these force equations to analyze overall force along each axis on the whole structure. We got net force as zero by adding all force components along the $\mathrm{x}$-axis of all nodes as follows:

$$
\begin{aligned}
& \lambda\left(\mathrm{L}_{b}\right)+\gamma\left(L_{d}-2 L_{b}\right)+\lambda\left(-L_{b}\right)+\gamma\left(-L_{d}+2 L_{b}\right)+\lambda\left(L_{b}\right)+\gamma\left(L_{d}-2 L_{b}\right)+\lambda\left(-L_{b}\right) \\
& +\gamma\left(-L_{d}+2 L_{b}\right)+\gamma\left(L_{b}-2 L_{d}\right)+\gamma\left(L_{b}-2 L_{d}\right)+\gamma\left(-L_{b}+2 L_{d}\right)+\gamma\left(-L_{b}+2 L_{d}\right)=0
\end{aligned}
$$

Similarly, we get net force as zero by adding all force components along the y-axis of all nodes as follows

$$
\begin{aligned}
& \gamma\left(L_{b}-2 L_{d}\right)+\gamma\left(L_{b}-2 L_{d}\right)+\gamma\left(-L_{b}+2 L_{d}\right)+\gamma\left(-L_{b}+2 L_{d}\right)+\lambda\left(L_{b}\right)+\gamma\left(L_{d}-2 L_{b}\right)+ \\
& \lambda\left(-L_{b}\right)+\gamma\left(-L_{d}+2 L_{b}\right)+\lambda\left(L_{b}\right)+\gamma\left(L_{d}-2 L_{b}\right)+\lambda\left(-L_{b}\right)+\gamma\left(-L_{d}+2 L_{b}\right)=0
\end{aligned}
$$

Again, we get net force as zero by adding all force components along the z-axis of all nodes as follows 


$$
\begin{aligned}
& \gamma\left(L_{b}-2 L_{d}\right)+\gamma\left(L_{b}-2 L_{d}\right)+\gamma\left(-L_{b}+2 L_{d}\right)+\gamma\left(-L_{b}+2 L_{d}\right)+\lambda\left(L_{b}\right)+\gamma\left(L_{d}-2 L_{b}\right)+ \\
& \lambda\left(-L_{b}\right)+\gamma\left(-L_{d}+2 L_{b}\right)+\lambda\left(L_{b}\right)+\gamma\left(L_{d}-2 L_{b}\right)+\lambda\left(-L_{b}\right)+\gamma\left(-L_{d}+2 L_{b}\right)=0
\end{aligned}
$$

It can be analyzed by the above calculations that all internal forces exerted by rigid bars and flexible strings acting on the whole structure have no net effect along any axis, which results in the structure to remain in equilibrium, this null result of all internal forces of members on whole structure shows the all force equations developed for each node are valid.

\section{Conclusion}

We defined the structure of the six-bar tensegrity ball structure robot and developed its nodes matrix. Force equations for each node were developed individually. The overall net effect of forces applied by strings and bars of the structure was investigated, and it was observed that forces applied by all strings and bars on the whole structure balance each node and form a whole spatial balanced structure.

\section{ACKNOWLEDGEMENT}

This research has been sponsored by the National Natural Science Foundation of China (Grant No. 51605111, 51675114, 51875111).

\section{NOMENCLATURE}

$L_{b}$ Length of each bar

$L_{d}$ Distance between each couple of parallel bars

$F_{b}$ internal force of bars

$T_{s}$ Internal force of strings

$\lambda$ Bar force density $\gamma$ String force density in group I strings after

\section{References}

[1] J. Yin, J. Duffy, and R. Crane ," An analysis for the design of self-deployable tensegrity and reinforced tensegrity prisms with elastic ties". 2002. 17(1): p. 38-45.

[2] Fuller R B, Tensile integrity structures, U.S.P. Office, Editor. 1959: United States.

[3] Otto F, Tensile structures. 1967, Cambridge: MIT Press.

[4] Fuller R B and Applewhite E J, Synergetics : explorations in the geometry of thinking. 1975, New York: Macmillan.

[5] R. Tobie, Pratt Institute, NY, A report on an inquiry into the existence, formation and representation of tensile structures. 1976.

[6] R. Motro, Tensegrity : from Art to Structural Engineering. 2011.

[7] Sultan C, Chapter 2 Tensegrity: 60 Years of Art, Science, and Engineering, in Advances in Applied Mechanics. 2009, Elsevier. p. 69145.

[8] J. Cai and J. Feng, Form-finding of tensegrity structures using an optimization method. Engineering Structures, 2015. 104: p. 126132.

[9] B. Fuller, "Tensegrity" Portfolio Art News Annual. 1961. 4: p. 112-127.

[10] K. Snelson, Continuous tension, discontinuous compression structures, U.S.P. 611, Editor. 1965: U.S.A.

[11] K.Snelson, " Kenneth Snelson, Art and Ideas," Kenneth Snelson, Marlborough Gallery, ,New York. 2013.

[12] C.R. Calladine, Buckminster Fuller's "Tensegrity" structures and Clerk Maxwell's rules for the construction of stiff frames. International Journal of Solids and Structures, 1978. 14(2): p. 161-172.

[13] D.S. Levin, http://biotensegrity.com

[14] G. Scarr, Biotensegrity. 2014: Handspring Publishing, United Kingdom.

[15] S. Levin, "The tensegrity-truss as a model for spine mechanics: biotensegrity". Journal of 
mechanics in medicine biology, 2002. 2(03n04): p. 375-388.

[16] R. Noguera, O.A. Nieto, and I. Tadeo, Extracellular matrix, biotensegrity and tumor microenvironment. An update and overview. 2012.

[17] Scarr. G, Biotensegrity: What is the big deal? Journal of Bodywork and Movement Therapies, 2020. 24(1): p. 134-137.

[18] S.H. Juan, and R.E. Skelton. "Dynamically stable collision avoidance for tensegrity based robots". in 2009 ASME/IFToMM International Conference on Reconfigurable Mechanisms and Robots. 2009. IEEE.

[19] M. Azadi, S. Behzadipour, and M. Faulkner, "Variable Stiffness Spring Using Tensegrity Prisms". 2010. 2(4): p. 041001.

[20] Y. Sugiyama and R. Hirai," Crawling and jumping by a deformable robot". 2006. 25(56): p. 603-620.

[21] K. Kim , A.K. Agogino and A.M. Agogino, Rolling Locomotion of Cable-Driven Soft Spherical Tensegrity Robots. Soft Robotics, 2020.

[22] Tibert G, "Deployable tensegrity structures for space applications", in Department of Mechanics. 2002, KTH Roval Institute of Technology: Sockholm, Sweden.

[23] K.Garanger, R. Miriam ,K.Matthew, Soft Tensegrity Systems for Planetary Landing and Exploration. arXiv preprint:2003.10999, 2020.

[24] A. Luo, R. E. Skelton and H. Liu. "Structure of the ball tensegrity robot." in 2014 IEEE
International Conference on Robotics and Biomimetics (ROBIO 2014). 2014. IEEE.

[25] A. Luo and H. Liu, "Analysis for Feasibility of the Method for Bars Driving the Ball Tensegrity Robot". Journal of Mechanisms and Robotics, 2017. 9(5): p. 051010-0510106.

[26] A. Luo, H. Liu and Y. Liu. "Analyzing the driving method for the ball tensegrity robot". in 2016 IEEE International Conference on Robotics and Biomimetics (ROBIO). 2016.

[27] A. Luo, H. Xin, P. Cao, "Motion simulation of six-bar tensegrity robot based on adams. in 2016" IEEE International Conference on Mechatronics and Automation. 2016. IEEE.

[28] SunSpiral V, George G, Bruce J , "Tensegrity based probes for planetary exploration: Entry, descent and landing (EDL) and surface mobility analysis". International Journal of Planetary Probes, 2013. 7: p. 13.

[29] Vespignani M, and Friesen J," Design of SUPERball v2, a Compliant Tensegrity Robot for Absorbing Large Impacts". 2018 Ieee/Rsi International Conference on Intelligent Robots and Systems, ed. A.A. Maciejewski, et al. 2018, New York: IEEE. 2865-2871.

[30] R.E. Skelton and M.C. de Oliveira, Tensegrity systems. Vol. 1. 2009: Springer.

[31] C.D.Crane , J. Duffy, and J.C. Correa, Static Analysis of Tensegrity Structures. Journal of Mechanical Design, 2005. 127(2): p. 257268. 